\title{
Towards a Methodology for Managing Competencies in Virtual Teams - A Systemic Approach
}

\author{
Marinita Schumacher, Julie Stal-Le Cardinal, and Jean-Claude Bocquet \\ Ecole Centrale Paris, Industrial Engineering Department, \\ Grande Voie des Vignes, 92295 Chatenay Malabry Cedex, France \\ \{marinita.schumacher, julie.le-cardinal, \\ jean-claude.bocquet\} @ecp. fr
}

\begin{abstract}
Virtual instruments and tools are future trends in Engineering which are a response to the growing complexity of engineering tasks, the facility of communication and strong collaborations on the international market. Outsourcing, off-shoring, and the globalization of organisations' activities have resulted in the formation of virtual product development teams. Individuals who are working in virtual teams must be equipped with diversified competencies that provide a basis for virtual team building. Thanks to the systemic approach of the functional analysis our paper responds to the need of a methodology of competence management to build virtual teams that are active in virtual design projects in the area of New Product Development (NPD).
\end{abstract}

Keywords: Virtual teams, competence management, methodology, systemic approach, house of quality.

\section{Introduction}

An integral part of fostering new knowledge, continued innovation and technological progress are improvements of the NPD and project management process [16]. As prospective partners of design projects are spread out over countries, organisations need access to worldwide communication to aspire worldwide competitiveness. In view of the increasing de-centralisation and globalisation of work processes, many organisations have responded to their dynamic environments by introducing virtual teams [6]. These virtual teams have unique characteristics including geographic distance, language and cultural barriers [17]. We are following the definition of Griffith who declares that a virtual team consists of Individuals that act interdependently through technology to achieve a common goal [7].

The changing nature of teams has brought on a need to identify the competencies that are necessary to work effectively in a virtual team environment. Competence is seen as the basis of competitiveness, it enables a company to offer products and valued services to customers and to generate new innovative products and services. Indeed this "new" way of considering human resources requires a more precise formalisation of concepts like competence or skills, in order to be able to identify the competencies needed to work effectively in a virtual team environment and to link employees efficiently to tasks. We refer to North who declaims that competencies are 
substantiated in the moment of knowledge application, which means that competencies only exist when the knowledge meets a task [14].

Broadly speaking, competence management comprises identification, acquisition, development, distribution, preservation and use of competencies and is the way in which organisations manage the competencies of the organisation, teams and individuals [19]. In our research we are essentially focussing on gaps between existing competencies and required competencies for current or future needs. In this case competence management occurs in the moment where a task and its required competencies are linked to an Individual and his acquired competencies [19] According to Harzallah and Vernadat, this is one of the major benefits of a competence management system for virtual teams: Individuals can be matched with specific jobs or tasks [8]. Competence matching is invaluable in the field of NPD that comprises numerous knowledge intensive tasks, and thus the need for highly skilled employees. One of the strategic reasons for virtual teams is to combine different competencies of experts from different locations to take advantages of market opportunities. Due to costs, such experts are a rare resource which has to be applied in order to achieve innovative products and thereby to accomplish competitive advantages. Nevertheless Olsen et al. [15] point out that only little empirical work is done on specific competencies related to product development and although virtual collaborative environments and platforms are getting more and more popular in various domains such as R\&D, problemsolving task forces, NPD, customer services etc., Horvarth and Tobin [11] distinguish that empirically-based prescriptions, guidelines and specifications for virtual team competencies are often missing. In our study we aim to respect the virtual aspect in NPD processes that claim other demands than traditional design projects in the area of NPD.

\section{Objectives and Framework of Our Research}

Our focus is set in providing a generic methodology of competence management for building virtual teams that are active in design projects in the area of NPD. This methodology should handle competencies in a virtual environment and should be adaptable to each specific organisational context. As distributed development projects can be difficult to control from a project management perspective, we aim to provide virtual teams whose competencies match the best with the requirements of design projects or by providing an individual who matches the best with a specific task to improve the NPD process.

The framework of this paper is organised into four stages: The following section 3 overviews in a first step our previous work based on the functional analysis, its objectives and main components that build the basis of our research. This section explains the systemic approach of the functional analysis that we choose to provide our methodology. In a second step we present the tool of the House of Quality that is part of the Quality Function Deployment [10]. The House of Quality and its adaption to our work is the focus of this study and bestride the results of section 4 . Detected key functions of the functional analysis are analysed in terms of existing techniques and 
concepts of competence management and virtual team building. Finally, section 5 contains the concluding remarks and perspectives for future work.

\section{A Systemic Approach}

In order to reach our goal, a competence management methodology for virtual team building, we chose the holistic approach of the functional analysis to get information from a systemic point of view. We regard our methodology as a system that interacts with all its components. The functional analysis helps to emphasize the importance of all the properties of a given system that is considered as a whole. The system cannot be determined or explained by its components alone but only by the interdependencies of each component that interacts constantly with the others [21]. With this approach we aim to identify needs and requirements and show interrelations of the system and its properties to establish sustainable research results.

The functional analysis aids to gain a clear picture about functionalities and usability of a new product or services and assists in identifying main actors. Referring to the APTE $®$ formalism for conducting a Value Analysis the functional analysis takes into consideration the various points of view of different research domains as well as the environmental aspects influencing a system [2].

\subsection{Functional Analysis}

Due to the systemic approach our "methodology of competence management for virtual team building" is considered a system that is described by functions. The functional analysis is performed to establish the system's functions, to define key functions related to the system and to control the distribution and maintenance of these functions in a systematic and useful manner [22].

We pursue the following steps that are presented in figure 1.

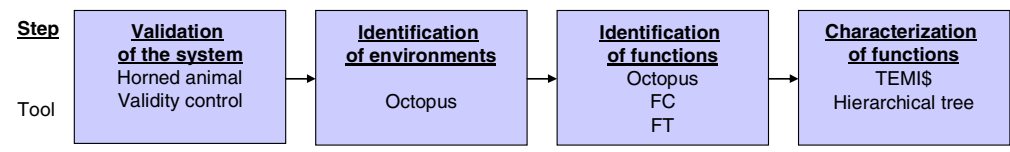

Fig. 1. Steps and tools of the functional analysis according to our study [18]

The specific results and their corresponding steps and tools are briefly described in this section. The tool of the horned animal helped us to get a first impression about the system itself, its target group, application areas and limits, the reason for its existence and its intention. After this step we passed a validity control to analyse the cause of the system, the objectives it aims for, and the risks of evolution or disappearance of the need. Through a tool that is called octopus we defined in a multidisciplinary brainstorming process 11 main environments and 40 sub-environments that permit us to take different concepts, critical terms and conditions into consideration. They are shown on the left side of following figure 2 . 


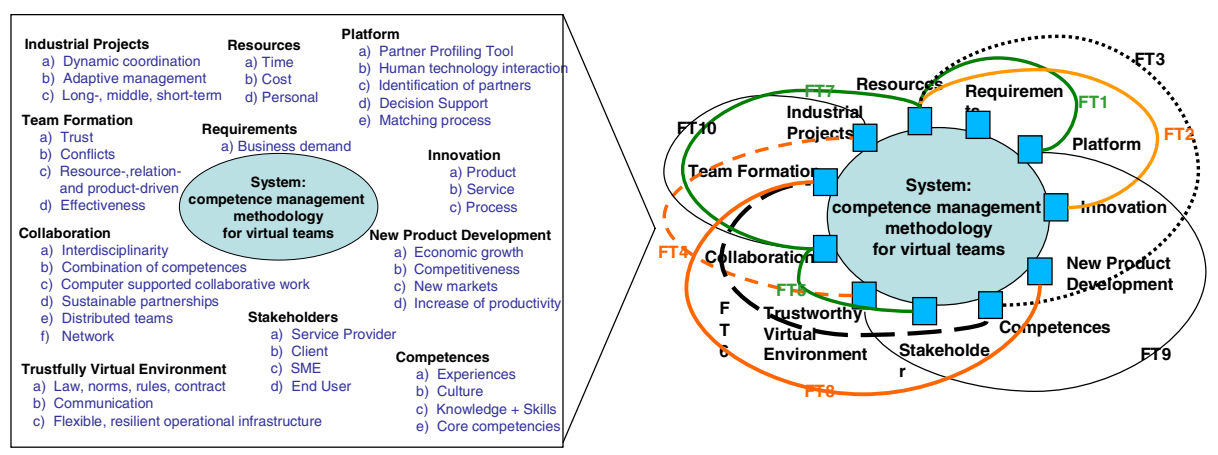

Fig. 2. Environments and extract of functions according to them [18]

The right side of the figure visualises an extract of the identification of functions according to the identified environments. In visual terms, the sub-clusters are not listed in this figure. We identified 243 transfer functions (FT) and 38 constraint functions (FC) by regarding each component of the system that interacts constantly with its environment. Transfer functions include at least two different environments that interact by the means of the system while constraint functions are generated by only one environment [2]. An example of FT and FC functions is shown in the following listing.

FT20 The system should be applicable to a wide range of organisations in the domain of NPD, to various application domains and to different design projects.

FC13

The system should provide that employees offer voluntary their individual competencies and their availability.

In a collaborative negotiation process we defined 40 key functions that represent main aspects of the system. They describe the optimum behaviour of the system and its terms of usability. Each key function is characterized with quantitative data in term of Time, Energy, Material, Information and Costs with a generic tool called TEMI\$ in the last step of the functional analysis. TEMI\$ supports the characterization of functions in a global way and help us to provide a definition of main quantitative aspects of the functions [18].

Based on the key findings, in the final step of our functional analysis the focus lays on the qualitative characterization of the functions. The importance of the key functions is measured in terms of percentages with the tool of the "hierarchical tree".

The tree structure provides a clear visibility of the large number of functions making up the system. It helps us to measure the importance of the functions in a qualitative way and to represent the system in a hierarchical form and to formulate substantiated qualitative recommendations. To determine the different basic categories of the top level of the hierarchical tree we referred to a model called CEISAR Enterprise Architecture Cube [4] [5]. 


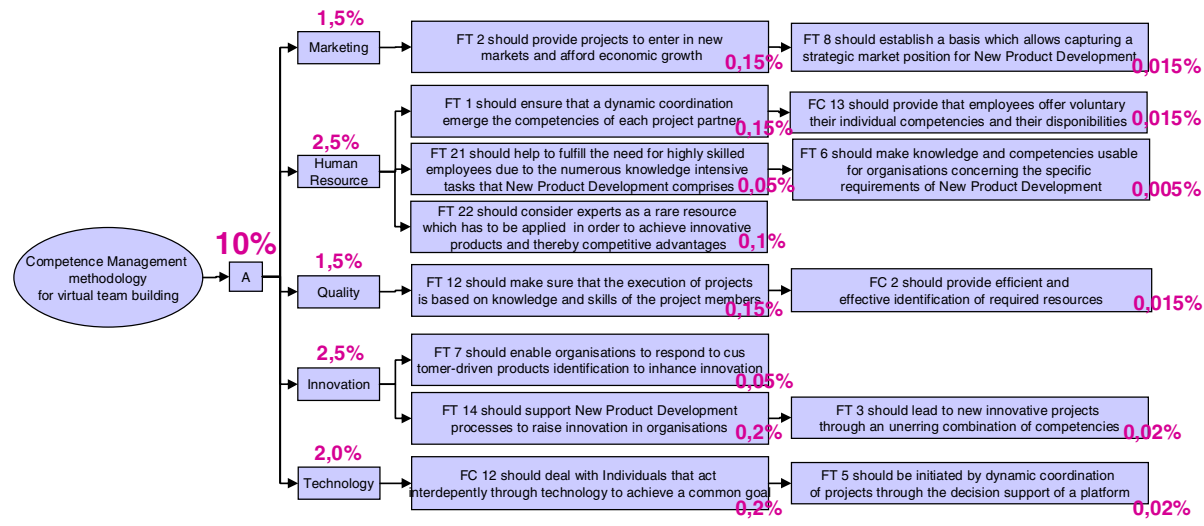

Fig. 4. Extract of branch A "I do" of the hierarchical tree according to our system [20]

We translated its eight mini-cubes to the foundation of our hierarchical tree and built eight categories A-F. Figure 5 gives a brief inside of the category A "I do" that is visualized as branch of the hierarchical tree according to the functions.

For reasons of visualisation this figure shows only the first and second level of functions. In the holistic figure of the hierarchical tree, all branches are treated and functions are broken down to several levels. The tree structure provides a clear visibility of the large number of functions making up the system. The hierarchical form delivers insights of the functions' importance. They depend on the specific needs of an organisation or also a particular design project without a real organisational structure which copes with the generic aspect of our methodology. It allows for the adaptation of not only each kind of organisation but also of each kind of design project. The percentages that are seen in figure 5 are just noted as examples. They depend on the purpose of the specific needs and have an important impact on the house of quality that is presented in the following section.

\subsection{House of Quality}

In a next step the detected key functions are analysed with a tool called "House of Quality" in terms of existing techniques and concepts of Competence Management and virtual team building. The House of Quality is a graphic tool for defining the relationship between customer requirements and the product capabilities that is a part of the Quality Function Deployment (QFD) [1]. QFD is a method to transform user demands into design quality and to set up the characteristics forming quality [10] Its ability to be adapted to the requirements of a particular problem makes it a very strong and reliable tool to use. It translates customer requirements called "Whats" and is based on our functions of the functional analysis, into a pertinent number of engineering targets, called "Hows". Its basic construction is made up of six major building blocks that compose the form of a house. These include customer requirements, technical requirements, a planning section, that deals with the importance of the costumers' requirements, a relationship matrix, a technical correlation matrix, and a 


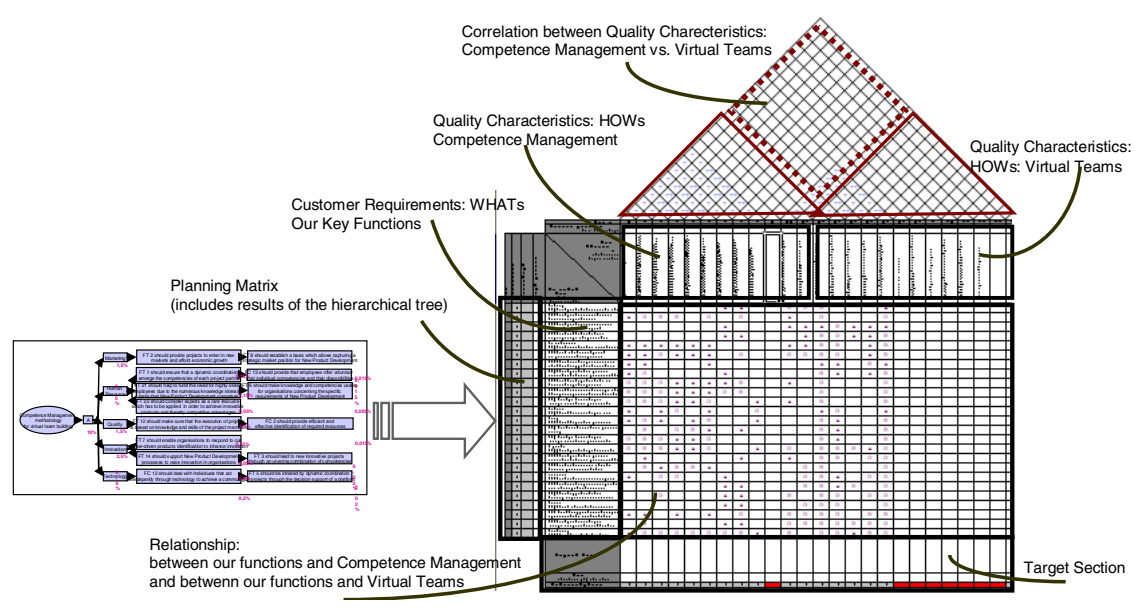

Fig. 5. House of Quality according to our system

technical priorities and targets section. The basic structure is a table with "Whats" on the left and "Hows" on the right site. The roof is composed as diagonal matrix of "Hows vs. Hows" and the body of the house builds a matrix of "Whats vs. Hows". Figure 6 gives an overview of the house of quality. It shows also the link between the hierarchical tree and the house of quality that is adapted to our study.

We use the tool of the House of Quality to deduce substantiated recommendations of our key functions that are detected by the functional analysis. Those essential functions are used as customers' requirements in this tool, visualised in figure 6 as "customer requirements". They describe what must be achieved to satisfy these demands. By putting them in relation with the technical requirements, the "Quality Characteristics", we try to get information about if they match their demand and to what degree. The main purpose of the relationship matrix is to establish a connection between the customer's product requirements and the performance measures designed to improve the product. The planning matrix shows the weighted importance of each requirement that our methodology is attempting to fulfil. Customer ratings are allocated to each requirement. In our case, the already analysed importance, presented as percentages of the hierarchical tree, will be incorporated into the planning matrix to get a coherent model. The customer ratings are combined with the weighted performance of each demand to produce an overall performance measure.

In this study we are concentrating on the relationship matrix and the correlation matrix. On the one hand we aim to find out strengths and weaknesses of the existent techniques and concepts due to the demand of the functions that could be adapted easily to our methodology. On the other hand through this approach we try to get information about those functions whose demands are apparently difficult to achieve. We intend to find out if the sum of our essential functions is satisfied by already existent techniques and concepts or if there are still solutions missing in the literature to satisfy our functions. With the help of the correlation matrix we try to get an idea if those quality characteristics have a specific rapport. 


\section{Results}

Our detected key functions of the functional analysis are put in relation to the technical requirements that we have detected by analysing existent techniques and concepts of competence management and virtual team building.

We used concepts and techniques defined in different already well-developed methodologies. These techniques have then been critically analysed in the context of our functions and analysed in the relationship matrix in terms of meeting their demands. The concepts used for the House of Quality are based on mainly two sources, one that focuses on competence management, and one that concentrates on virtual team building. They are briefly explained in the following listing.

1. CRAI (Competency Resource Aspect Individual) approach that provides a formal representation of acquired and required individual competencies and define competencies in a generic way [3] [9].

2. Product Development 2.0 that provides a collaborative Web 2.0 environment for NPD that changes the structure of team interaction and collaboration from a "push" to a "pull" system [12] [13].

Following figure 7 gives an extract of the results of our house of quality. Competence management and virtual teams are each presented with an extract of five quality characteristics. They are analysed in terms of the relationship to the two beforehand described functions FT 20 and FC 13. Furthermore we are concentrating on the upper part of the house of quality to give a brief insight of the correlation between the quality characteristics of competence management and virtual teams.

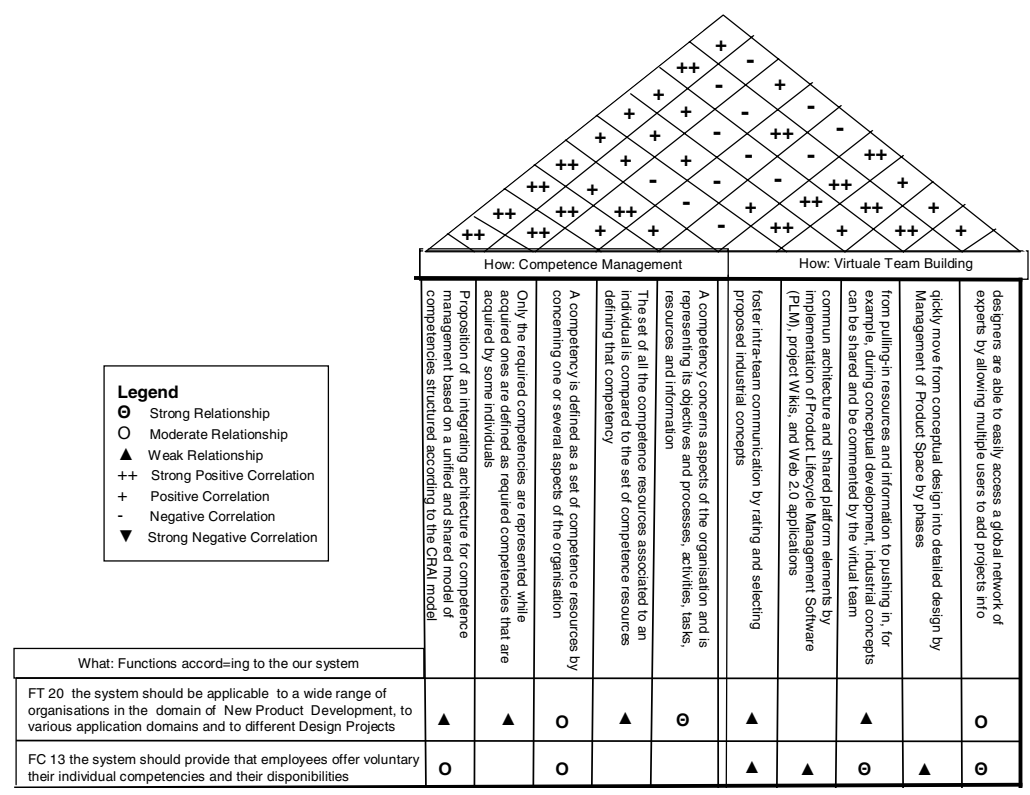

Fig. 6. Extract of Results according to our House of Quality 
For reasons of visualisation this figure shows only a brief extract of our house of quality. We have analysed more than 50 different concepts and the list is not yet finished. The house of quality helps us to get a clear idea about which concepts are compatible and for which ones we have to find other solutions. On the one hand, the results of this critical analysis point out strengths and weaknesses of the existent techniques and concepts due to the demand of the functions that could be adapted easily to our methodology. On the other hand, this approach gives information about those functions whose demands are apparently difficult to achieve. Some functions are easily achievable, while others are complex and difficult to meet.

The structure of the house of quality seems especially useful for us as we are in the process of the initial decomposition and structuring of functional requirements. As a result of a successful analysis with this tool, we get substantiated information of our key functions that help us to interpret them and to provide guidelines for our methodology. A brief extract of those outcomes is seen in the following listing.

- The end of our dynamic system of methodology of competence management for virtual teams should be the realisation of the conception of the innovative product or service and not its industrialisation.

- Within the competence management methodology competence ontologies should be stored together with process descriptions and the process flows of the design projects. This competence approach should differentiate between competencies in terms of cognitive knowledge, procedural (methodical) knowledge on and social and competencies derived from activities.

- Moreover, competence should be described qualitatively and quantitatively. The qualitative evaluation differentiates the degree of expertise in "no competence", "basic competence", "independent work on the task" and "expert". Quantitative evaluation asks for the frequency of certain activities in a design process or in relation to a defined time frame.

- Characteristics of individual competencies as well as characteristics of the tasks and their activities for a new design are imprecise data that are hard to measure. Our methodology of competence management should be capable of dealing with this challenge.

- It should be connected on long term to the HR strategy of an organisation that could include assessment, recruitment, promotion, training, coaching, and evaluating processes.

Before having used this method, we did not have a clear picture of the different concepts that help to satisfy the specific functions of our system. In our example, the tool of the house of quality helped us to take our key functions in relation to existent concepts into consideration and to find substantiated guidelines. Furthermore, the correlation matrix helped us to analyse which quality characteristics are compatible to provide our methodology of competence management for virtual team building.

\section{Outlook and Perspectives}

In further research we aim to determine our "methodology for competence management for virtual team building" by proposing other techniques or concepts that satisfy 
all the functions of our system or at least the critical ones. We aim to find out if the sum of our essential functions is satisfied by already existent techniques and concepts or if there are still solutions missing in the literature to satisfy our functions. We will focus our work on the still missing solutions to cope the demand of those functions. Firstly, we used concepts defined in different already well-developed methodologies, secondly we aim to use concepts that we find in the practical field.

After determining which functions are most important to the organisation of a specific design project, the quality characteristics must be translated into particulate specifications. We aim to give more detailed recommendations for the specific quality characteristics. Nothing can be produced, serviced or maintained without detailed specifications or some set of given standards. That is the reason why our objective is to provide a catalogue of precise possibilities of HOWs. Another step will be to include the percentages of importance of the hierarchical tree to have a clear idea of the significance of each function.

In further research we aim to determine the formalisation of our "methodology for competence management for virtual team building". It should improve the NPD process by providing a virtual team whose competencies match the best to the requirements of design projects. In a proximate step our methodology must be applied to the industrial reality and especially to innovative organisations in the domain of new services, processes or product development. We intend to build virtual teams by matching individual competencies of potential team members to requirements of the design projects. It should be applicable to a wide range of organisations in the domain of NPD, to various application domains and to different design projects.

\section{References}

1. Akao, Y.: Quality Function Deployment. Productivity Press, Portland (1990)

2. Apte, La Méthode APTE (®) d'AV/AF, Petrelle (2000)

3. Berio, G., Hazallah, M.: Towards an integrating architecture for competence management. Computer in Industry 58, 199-209 (2007)

4. Ceisar, ECP - Center of Excellence in Enterprise Architecture, White Paper, Business Process Modeling (April 2008), http: / / www . ceisar.org (dated: 06/04/2008)

5. Ceisar, ECP - Center of Excellence in Enterprise Architecture, White Paper, Enterprise Modelling (April 2008), http: / / www . ceisar .org (dated: 29/04/2008)

6. Grenier, R., Metes, G.: Going virtual: Moving our organsiation in the 21st Century. Prentice Hall, Upper Saddle River (1995)

7. Griffith, T.L., Sawyer, J.E., Neale, M.A.: Virtualness and Knowledge in Teams: Managing the Love Triangle. Organizations, Individuals, and Information Technology, MIS Quarterly (27), 265-287 (2003)

8. Harzallah, M., Vernadat, F.: Human resource competency management in enterprise engineering. In: 14th IFAC World Congress of Information Control in Manufacturing, Beijing, China (1999)

9. Harzallah, M., Vernadat, F.: IT-based competency modeling and management: from theory to practice in enterprise engineering and operations. Computers in Industry 48, 157-179 (2002)

10. Hauser, J., Clausing, D.: House of Quality. Harvard Business Review (2009) 
11. Horvarth, L., Tobin, T.J.: Twenty-first century teamwork: Defining competencies for virtual teams. Virtual Teams 8, 239-258 (2001)

12. Marion, J.T., Schumacher, M.: Moving New Venture NPD from Information Push to Pull Using Web 2.0. Submitted for International Conference on Engineering Design, ICED 2009, Stanford (2009)

13. Marion, T.J.: NPD Practices at Early-Stage Firms: A Pilot Study. Academy of Management 2008, Annual Meeting, paper 13663 (2008)

14. North, K.: Wissensorientierte Unternehmensführung - Wertschöpfung durch Wissen, 3rd edn. Gabler Verlag, Wiesbaden (2002)

15. Olsen, R.J., Harmsen, H., Friis, A.: Linking quality goals and product development competences. Food Quality and Preference 19, 33-42 (2008)

16. Ramesh, B., Tiwana, A.: Supporting Collaborative Process Knowledge Management in NPD Teams. Decision Support Systems 27, 213-235 (1999)

17. Rezgui, Y.: Exploring virtual team-working effectiveness in the construction sector. Interacting with Computers 19, 96-112 (2007)

18. Schumacher, M., Le Cardinal, J., Mekhilef, M.: A competence management methodology for virtual teams - A systemic approach to support innovation processes in SME's. In: International Design Conference: Design 2008, Dubrovnik (2008)

19. Schumacher, M., Le Cardinal, J., Mekhilef, M.: Competence management for virtual team building: A survey. In: International Conference on Integrated, Virtual and Interactive Engineering for fostering Industrial Innovation - IDMME 2008, Peking (2008)

20. Schumacher, M., Le Stal-Cardinal, J., Bocquet, J.C.: Towards a generic methodology of virtual team building adapted to specific needs of design projects, Cahier d'études et de recherche- ECP-2009-02 (2009)

21. Snodgrass, T.J.: Function Analysis - The Stepping Stones to Good Value, CVS at KASI. Muthiah, CVS (1986)

22. Yosida, K.: Functional analysis, 5th edn. Springer, Heidelberg (1978) 